\title{
Caracterización de actinomicetos de sedimento marino y su actividad antagonista frente a Vibrio sp. aislados de «langostino blanco» Litopenaeus vannamei (Boone, 1931)
}

\author{
CHARACTERIZATION OF ACTINOMYCETES OF MARINE SEDIMENT AND THEIR ANTAGONISTIC \\ ACTIVITY AGAINST VIBRIO SP. ISOLATED FROM «WHITELEG SHRIMP» \\ Litopenaeus vannamei (BOONE, 1931)
}

\author{
Ulrike Tarazona J. ${ }^{1}$, Jorge León $\mathbf{Q .}^{1,3}$, Nadia Galindo C. ${ }^{1}$, Marisol Vallejo ${ }^{2}$, \\ Emilio Marguet ${ }^{2}$
}

\section{Resumen}

\begin{abstract}
El objetivo principal del presente estudio fue evaluar la actividad antagonista de una colección de 24 actinomicetos de sedimento marino frente a especies silvestres de Vibrio. Los actinomicetos fueron caracterizados por su crecimiento en Agar Marino y su actividad enzimática extracelular sobre diferentes sustratos. La actividad antagonista se evaluó mediante pruebas in vitro frente a seis cepas de Vibrio aisladas de Litopenaeus vannamei. Los actinomicetos de mayor antagonismo fueron evaluados por su crecimiento en medios estándares ISP y se hicieron observaciones de microfotografías por técnicas de microscopía electrónica de barrido (MEB). Las cepas de Vibrio fueron evaluadas e identificadas mediante técnicas bioquímicas convencionales y confirmadas por PCR. Los resultados indicaron que la totalidad de actinomicetos mostraron al menos alguna actividad multi-enzimática extracelular, predominando las que hidrolizan almidón (79\%). Las pruebas de antagonismo señalaron que el 54\% (13/24) de actinomicetos tuvieron actividad anti-Vibrio, siendo las cepas M10-77 y M11-116 (B) las que produjeron los halos de inhibición de mayor tamaño. Por otro lado, la identificación basada en secuencia del gen 16S ARNr corroboró la relación de las cepas aisladas de L. vannamei con el género Vibrio; asimismo, algunas de estas actinobacterias fueron identificadas como Streptomyces.
\end{abstract}

Palabras clave: probióticos; antagonistas; acuicultura; Vibrio; langostino blanco

\footnotetext{
${ }^{1}$ Facultad de Ciencias Biológicas, Universidad Nacional Mayor de San Marcos, Lima, Perú

${ }^{2}$ Facultad de Ciencias, Universidad Nacional de la Patagonia San Juan Bosco, Trelew, Argentina

${ }^{3}$ E-mail: jleonq@unmsm.edu.pe
}

Recibido: 24 de octubre de 2017

Aceptado para publicación: 16 de febrero de 2018 
The aim of the present study was to evaluate the antagonistic activity of a collection of 24 actinomycetes of marine sediment against wild species of Vibrio. Actinomycetes were characterized by their growth in Marine Agar and their extracellular enzymatic activity on different substrates. The antagonist activity was determined by in vitro tests against six strains of Vibrio isolated from Litopenaeus vannamei. Actinomycetes with greater antagonism were evaluated by their growth in standard ISP media and observations of microphotographs by scanning electron microscopy (SEM) techniques were done. The Vibrio strains were evaluated and identified by conventional biochemical techniques and confirmed by PCR. The results indicated that all actinomycetes showed at least some extracellular multi-enzymatic activity, predominating those that hydrolyze starch (79\%). Antagonism tests indicated that 54\% (13/24) of actinomycetes had anti-Vibrio activity, being the strains M10-77 and M11-116 (B) those that produced the largest inhibition halos. On the other hand, the sequence-based identification of the $16 \mathrm{~S}$ rRNA gene corroborated the relationship of the isolated strains of L. vannamei with the genus Vibrio; likewise, some of these actinobacteria were identified as Streptomyces.

Key words: probiotics; antagonists; aquaculture; Vibrio; white shrimp

\section{INTRODUCCIÓN}

Litopenaeus vannamei (Boone, 1931), «langostino blanco», es una de las especies más importantes en la actividad acuícola del Perú. El tipo de cultivo de esta especie crea condiciones artificiales que favorecen la selección, adaptación y crecimiento de comunidades bacterianas que forman parte de la microbiota normal de organismos acuáticos como Vibrio, Pseudomonas, Aeromonas, Plesiomonas, Flavobacterium y otros (Liu et al., 2014). Estas comunidades no representan riesgo alguno para los crustáceos a menos que se encuentren estresados, débiles o inmunodeprimidos (You et al., 2005). En los últimos años han surgido problemas de enfermedades infecciosas y el deterioro de condiciones ambientales que afectan severamente a la producción del langostino blanco, mayormente causados por bacterias de los géneros Vibrio y Aeromonas (Liu et al., 2014).

El uso de probióticos ha demostrado ventajas en la producción controlada de organismos acuáticos, en especial en las eta- pas de su desarrollo larval y juvenil. Probióticos formulados con base a Lactobacillus spp y Bacillus spp son utilizados en cultivos de langostinos (Nimrat et al., 2012), así como ciertas cepas de Vibrio y Pseudomonas no patógenas (Verschuere et al., 2000); sin embargo, pocos estudios han reportado actinomicetos como posibles probióticos (Das et al., 2010).

Los actinomicetos marinos son bacterias filamentosas con múltiples capacidades metabólicas y fisiológicas, productoras de varios compuestos naturales, entre las que se encuentran sustancias con actividad inhibitoria, y que han sido propuestas como posibles agentes probióticos en la acuicultura marina (Das et al., 2010).

En el presente estudio se describen algunas pruebas in vitro que permiten evaluar y seleccionar cepas nativas de actinomicetos marinos con actividad antimicrobiana contra especies de Vibrio, como alternativa en el tratamiento de infecciones en $L$. vannamei. Asimismo, permite plantear su evaluación como posibles agentes probióticos y biorremediantes naturales en el marco de una práctica de acuicultura sostenible. 


\section{Materiales y Métodos}

\section{Litopenaeus vannamei}

Los especímenes de L. vannamei $(n=50)$ fueron donados por una empresa langostinera privada, situada en la región Tumbes, Perú. Se seleccionaron langostinos juveniles, moribundos y con signos aparentes de vibriosis.

\section{Cepas de Actinomicetos}

Se utilizaron 24 cepas de actinomicetos pertenecientes a la colección del Laboratorio de Ecología Microbiana de la Facultad de Ciencias Biológicas de la Universidad Nacional Mayor de San Marcos, localizada en Lima, Perú. Estas cepas fueron aisladas de sedimento marino colectados en la Bahía de Independencia (Reserva Nacional de Paracas, Perú) y Bahía de Ancón (Lima, Perú) y reportadas en estudios previos (León et al., 2007).

\section{Aislamiento de Vibrio spp}

El aislamiento se hizo a partir de especímenes enfermos de langostinos juveniles. Se preparó un «pool» de muestras a partir de órganos internos y luego fueron sembrados en el medio Agar Tiosulfato Citrato Bilis Sacarosa (TCBS) y Agar Tripticasa de Soya (TSA) con adición de $1 \%$ de $\mathrm{NaCl}$ (Yano et al., 2015). Los cultivos fueron incubados a $30^{\circ} \mathrm{C}$ por $48 \mathrm{~h}$. Las cepas aisladas fueron conservadas en el medio TSA + $1 \% \mathrm{NaCl}$.

\section{Identificación de las Cepas de Vibrio}

Las cepas presuntivas de Vibrio fueron caracterizadas e identificadas según las directrices del Manual de Bacteriología Sistemática Bergey's (Baumann y Schubert, 1984). Adicionalmente, fueron evaluadas por su capacidad de crecimiento en medios conteniendo $1.5,2,4,6,8$ y $10 \%$ de $\mathrm{NaCl}$. Todos los cultivos fueron incubados a $30^{\circ} \mathrm{C}$ por $24-48 \mathrm{~h}$.

\section{Antibiograma de las Cepas de Vibrio}

Se realizó según las pautas del Instituto Nacional de Salud (2002) con modificaciones (Agar Mueller Hinton con $1 \%$ de $\mathrm{NaCl}$ ). Se utilizaron discos de antibióticos de uso frecuente en la industria langostinera (Vaseeharan et al., 2005).

\section{Caracterización Fenotípica de Actino- micetos}

Las colonias de actinomicetos fueron caracterizadas en Agar Marino y la producción de enzimas extracelulares (EEC) en Agar Marino con adición de substratos (caseína, gelatina, celulosa, almidón, tween 80 , lecitina y urea), además de agar DNA (León et al., 2007). La observación microscópica se realizó a partir de «microcultivos» (Wang et al., 2014). En todos los casos, los cultivos fueron incubados a $28^{\circ} \mathrm{C}$ por 5-7 días. Las cepas de mayor actividad antagonista fueron caracterizadas además por su crecimiento en medios estándar ISP (International Streptomyces Proyect) y observaciones por microscopía electrónica de barrido (Inspect ${ }^{\mathrm{TM}}$ S50, FEI), cuyas muestras se procesaron según las pautas metodológicas de Colona et al. (2014).

\section{Ensayos in vitro de la Actividad Antago- nista de Actinomicetos}

Los actinomicetos fueron enfrentados a vibrios silvestres aislados en el presente estudio según la metodología descrita por León et al. (2007). Se utilizaron además como cepas controles a $V$. harveyi CECT $525^{\mathrm{T}}$ y V. alginolyticus CCM $2578^{\mathrm{T}}$. Los resultados del antagonismo se expresaron por el tamaño $(\mathrm{mm})$ de los halos de inhibición.

\section{Identificación Molecular de Vibrio y Actinomicetos Antagonistas}

La extracción de ADN de actinomicetos y vibrios se realizó con un kit de purificación (Wizard Genomic ADN Purification Kit Promega ${ }^{\circledR}$ ) siguiendo las instrucciones del 
fabricante. Para las amplificaciones del gen ARN ribosomal 16S se utilizaron cebadores universales 27F (5'-AGA GTT TGA TCC TGG CTC AG-3') y 1492R (5'-TAC GGY TAC CTT GTT ACG ACTT-3') bajo las siguientes condiciones: desnaturalización inicial $\left(94{ }^{\circ} \mathrm{C}\right.$ por $\left.2 \mathrm{~min}\right), 25$ ciclos de desnaturalización $\left(94^{\circ} \mathrm{C}\right.$ por $2 \mathrm{~min}$ ), alineamiento $\left(45^{\circ} \mathrm{C}\right.$ por $\left.1.5 \mathrm{~min}\right)$, extensión $\left(72^{\circ} \mathrm{C}\right.$ por $2 \mathrm{~min}$ ) y una extensión final $\left(72^{\circ} \mathrm{C}\right.$ por 3 min). Los productos de amplificación se secuenciaron mediante los servicios de Macrogen (Seúl, Corea). Para las amplificaciones de los vibrios se utilizaron los cebadores 518f (CCA GCA GCC GCG GTA ATA CG) y 800r (TAC CAG GGT ATC TAA TCC).

\section{Análisis Filogenético}

El alineamiento de las secuencias se realizó mediante el Programa Chromas Pro 1.7.6 (Technelysium Pty Ltd) y Clustal X 2.0 (Larkin et al., 2007). Los resultados se compararon con la base de datos del National Center for Biotechnology Information (NCBI) mediante el Programa Basic Local Alignment Search Tool (BLAST) (Altschup et al., 1990). Se construyeron los árboles filogenéticos (Neighbour-Joining Method) a partir de una matriz de distancias genéticas entre pares calculadas con el algoritmo de Jukes-Cantor (Jukes y Cantor, 1969). Todo el análisis se realizó usando el paquete MEGA 6 (Tamura et al., 2013).

\section{Resultados}

A partir del medio TCBS se aislaron seis cepas presuntivas del género Vibrio, que fueron codificadas como VP (aisladas de hepatopáncreas) y VL (aisladas de hemolinfa). La caracterización bioquímica de estas cepas se observa en el Cuadro 1. En todos los casos comparten características culturales de las cepas referenciales $V$. alginolyticus y $V$. parahaemolyticus.
Los estudios de halotolerancia permitieron observar el crecimiento de los vibrios en caldo marino conteniendo desde 1 hasta $8 \%$ de $\mathrm{NaCl}$ (Cuadro 2). El Cuadro 3 muestra el perfil antibiótico de las cepas de vibrios silvestres, indicando la alta sensibilidad frente a la mayoría de los antibióticos probados. Todas mostraron resistencia a Novobiocina (NOV), pero solo V3L, V5P3, V8P2 y V8P3 a Penicilina $\mathrm{G}(\mathrm{PEN})$.

Las características fenotípicas de los actinomicetos se encuentran en el Cuadro 4 . En general, las colonias fueron circulares, de bordes irregulares, elevados, convexos y secos, con hifas aéreas bien desarrolladas. El crecimiento en Agar Marino ocurrió entre 7 y 21 días. Las colonias variaron entre 7 y 20 $\mathrm{mm}$ de diámetro, la mayoría blanca o blancagrisácea, algunas con pigmentación (marrón, amarillo o rojizo) al reverso de la colonia.

El Cuadro 5 muestra la capacidad de los actinomicetos para producir EEC. E1 96\% presentó actividad proteolítica sobre la gelatina, el $78 \%$ amilolítica y $63 \%$ lipolítica sobre la lecitina. Asimismo, el 58\% fueron capaces de hidrolizar Tween 80 y otro tanto la caseína, mientras 38 y $33 \%$ hidrolizaron urea y DNA, respectivamente. Las cepas I434B y M11-116d resaltaron por su actividad hidrolítica sobre siete sustratos. Ninguna cepa mostró actividad sobre celulosa.

El Cuadro 6 muestra las características de los actinomicetos seleccionados [M10-77, M11-116(B), M11-116d (A), M11-116d (B) y I300A] en los medios ISP, donde se resalta a las colonias secas, pulverulentas y costrosas. Solo la cepa M10-77 mostró moderada pigmentación melanoide de color marrón verdoso y marrón oscuro en los medios ISP 6 e ISP 7 respectivamente. Con la tinción Gram se observaron filamentos finos y ramificados en su mayoría, así como Gram positivos con esporas ovaladas en cadena. Asimismo, los microcultivos permitieron observar filamentos delgados con gran canti- 
Cuadro1. Pruebas bioquímicas del género Vibrio aislados de langostinos y cepas referenciales*

\begin{tabular}{|c|c|c|c|c|c|c|c|c|c|c|}
\hline \multicolumn{3}{|c|}{ Pruebas bioquímicas ${ }^{1}$} & $\stackrel{m}{>}$ & $\hat{n}_{n}^{n}$ & $\stackrel{\Re}{\stackrel{n}{>}}$ & $\vec{\infty}$ & $\stackrel{\substack{\infty \\
>}}{>}$ & $\hat{\infty}_{>}^{\infty}$ & $\begin{array}{l}\underset{\pi}{*} \\
\underset{\sim}{>} \\
>\end{array}$ & 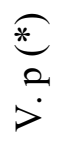 \\
\hline Catalasa & & & + & + & + & + & + & + & + & + \\
\hline Oxidasa & & & + & + & + & + & + & + & + & + \\
\hline TSI & & & $\mathrm{A} / \mathrm{A}$ & $\mathrm{A} / \mathrm{A}$ & $\mathrm{A} / \mathrm{A}$ & $\mathrm{A} / \mathrm{A}$ & $\mathrm{A} / \mathrm{A}$ & $\mathrm{A} / \mathrm{A}$ & $\mathrm{A} / \mathrm{A}$ & $\mathrm{K} / \mathrm{A}$ \\
\hline LIA & & & + & + & $\mathrm{nc}$ & - & - & + & + & + \\
\hline MR & & & + & + & + & + & + & + & + & + \\
\hline VP & & & - & - & - & - & - & - & - & - \\
\hline Citrato & & & - & + & + & + & - & - & - & - \\
\hline \multirow[t]{3}{*}{ MIO } & Motilidad & & + & + & + & + & + & + & + & + \\
\hline & Indol & & + & + & + & + & + & + & + & + \\
\hline & Ornitina & & + & + & + & + & + & + & + & + \\
\hline \multirow[t]{3}{*}{ SIM } & $\mathrm{H}_{2} \mathrm{~S}$ & & - & - & - & - & - & - & - & - \\
\hline & Indol & & + & + & + & + & + & + & + & + \\
\hline & Motilidad & & + & + & + & + & + & + & + & + \\
\hline \multirow{4}{*}{$\begin{array}{l}\text { Hugh \& } \\
\text { Leifson }\end{array}$} & Manosa & $\mathrm{O}_{2}$ & + & + & + & + & + & + & + & + \\
\hline & & $\operatorname{Sin} \mathrm{O}_{2}$ & + & + & + & + & + & + & + & + \\
\hline & Dextrosa & $\mathrm{O}_{2}$ & + & + & + & + & + & + & + & + \\
\hline & & $\operatorname{Sin} \mathrm{O}_{2}$ & + & + & + & + & + & + & + & + \\
\hline
\end{tabular}

TSI: Agar-hierro-triple azúcar; LIA: lisina hierro agar; MR: rojo de metilo; VP: Voges Proskauer; MIO: movilidad, indol, ornitina; SIM: sulfuro, indol, motilidad

+/-: positivo/negativo; nc: no creció; A: acidez; K: alcalinidad

VP: cepas aisladas de hepatopáncreas; VL: cepas aisladas de hemolinfa

$\left({ }^{*}\right)$ V. a.: V. alginolyticus, V. p.: V. parahaemolyticus

Cuadro 2. Halotolerancia de bacterias del género Vibrio aisladas de Litopenaeus vannamei

\begin{tabular}{lcccccc}
\hline \multirow{2}{*}{ Cepas } & \multicolumn{7}{c}{ Concentración de $\mathrm{NaCl}(\%)$} \\
\cline { 2 - 7 } & 1 & 2 & 4 & 6 & 8 & 10 \\
\hline V3L & +++ & +++ & +++ & +++ & ++ & ++ \\
V5P3 & +++ & +++ & +++ & +++ & +++ & +++ \\
V7P3 & ++ & ++ & ++ & ++ & ++ & ++ \\
V8P1 & +++ & +++ & +++ & +++ & +++ & ++ \\
V8P2 & +++ & +++ & +++ & +++ & ++ & - \\
V8P3 & +++ & +++ & +++ & +++ & +++ & +++ \\
V. alginolyticus & +++ & +++ & +++ & +++ & ++ & ++ \\
V. harveyi & ++ & ++ & ++ & + & + & + \\
\hline
\end{tabular}

Crecimiento: $(+++)$ : abundante, $(++)$ : moderado, $(+)$ : escaso, (-): no crecimiento

VP: cepas aisladas de hepatopáncreas; VL: cepas aisladas de hemolinfa

$\left({ }^{*}\right)$ Cepas referenciales 
Cuadro 3. Antibiograma de cepas del género Vibrio aisladas de langostinos con signos de vibriosis

\begin{tabular}{lccccccc}
\hline Antibiótico & $\begin{array}{c}\text { Concentración } \\
(\mu \mathrm{g})\end{array}$ & V5P3 & V3L & V8P3 & V7P3 & V8P1 & V8P2 \\
\hline Ácido nalidíxico (NAL) & 30 & $\mathrm{~S}$ & $\mathrm{~S}$ & $\mathrm{R}$ & $\mathrm{S}$ & $\mathrm{S}$ & $\mathrm{S}$ \\
Gentamicina (GM) & 10 & $\mathrm{I}$ & $\mathrm{I}$ & $\mathrm{S}$ & $\mathrm{S}$ & $\mathrm{S}$ & $\mathrm{I}$ \\
Amikacina (AK) & 30 & $\mathrm{R}$ & $\mathrm{R}$ & $\mathrm{S}$ & $\mathrm{S}$ & $\mathrm{S}$ & $\mathrm{R}$ \\
Penicilina G (PEN) & $10^{*}$ & $\mathrm{R}$ & $\mathrm{R}$ & $\mathrm{R}$ & $\mathrm{I}$ & $\mathrm{R}$ & $\mathrm{R}$ \\
Trimetroprim/sulfa (SXT) & 23.75 & $\mathrm{~S}$ & $\mathrm{~S}$ & $\mathrm{R}$ & $\mathrm{S}$ & $\mathrm{S}$ & $\mathrm{S}$ \\
& 11.25 & & & & & & \\
Ciprofloxacino (CIP) & 5 & $\mathrm{I}$ & $\mathrm{I}$ & $\mathrm{S}$ & $\mathrm{S}$ & $\mathrm{S}$ & $\mathrm{S}$ \\
Nitrofurantoina (NIT) & 300 & $\mathrm{~S}$ & $\mathrm{~S}$ & $\mathrm{R}$ & $\mathrm{S}$ & $\mathrm{S}$ & $\mathrm{S}$ \\
Novobiocina (NOV) & 5 & $\mathrm{R}$ & $\mathrm{R}$ & $\mathrm{R}$ & $\mathrm{R}$ & $\mathrm{R}$ & $\mathrm{R}$ \\
Estreptomicina (S) & 10 & $\mathrm{~S}$ & $\mathrm{~S}$ & $\mathrm{~S}$ & $\mathrm{~S}$ & $\mathrm{~S}$ & $\mathrm{~S}$ \\
Enroblend (EBD) & $5 \%$ & $\mathrm{~S}$ & $\mathrm{~S}$ & $\mathrm{~S}$ & $\mathrm{~S}$ & $\mathrm{~S}$ & $\mathrm{~S}$ \\
Florfenicol (FLD) & $2 \%$ & $\mathrm{~S}$ & $\mathrm{~S}$ & $\mathrm{R}$ & $\mathrm{S}$ & $\mathrm{S}$ & $\mathrm{S}$ \\
\hline
\end{tabular}

$\mathrm{R}$ : resistente, I: intermedio, S: sensible

* Unidades internacionales

dad de esporas individuales típicos de Streptomyces. Finalmente, las microfotografías de dos cepas seleccionadas [M11116d (B) y M10-77] permitieron observar hifas muy ramificadas, aunque con escasas esporas (Figura 1).

Las pruebas de antagonismo indicaron que el $71 \%$ de los actinomicetos tienen actividad inhibitoria en al menos de una cepa de vibrio silvestre. Asimismo, el 54\% de actinomicetos lograron inhibir al vibrio V8P3; sin embargo, solo las cepas M10-77 y M11116(B) inhibieron a seis cepas de vibrios más los controles $V$. alginolyticus, $V$. harveyii y V. parahaemolyticus. Cabe resaltar el antagonismo de M11-116d (B) y II334C frente a la cepa V8P3 con halos de inhibición de 22.9 y $28.4 \mathrm{~mm}$ de diámetro respectivamente (Cuadro 7).

La identificación molecular de las cepas de Vibrio señaló entre 99 y 100\% de homología con $V$. alginolyticus, $V$. parahaemolyticus, $V$. diabolicus y $V$. mytili
(Cuadro 8). Las cepas V3L, V5P3 y V8P2 forman un clado separado junto a Vibrio brasiliensis (Figura 2). Respecto a actinomicetos seleccionados, se lograron identificar como miembros del género Streptomyces (Figura 3), resultando las cepas B1TG1 y M11 105 como S. althioticus y S. albidoflabus, respectivamente. Asimismo, las cepas M11-116d y I434B se encuentran en un mismo clado, mostrando mayor homología con las especies $S$. variabilis y $S$. griseorubens, respectivamente.

\section{Discusión}

Especies de Vibrio en la acuicultura de Litopenaeus vannamei han sido investigados en los últimos años, siendo considerados como patógenos primarios Vibrio harveyi, $V$. parahaemolyticus y $V$. vulnificus (Zhou et al., 2012). En el presente estudio, si bien se logró aislar e identificar cepas de Vibrio de especímenes enfermos, no se les pudo confirmar como los causantes directos de la 
Cuadro 4. Caracterización fenotípica de 24 cepas de actinomicetos de sedimento marino

\begin{tabular}{llllc}
\hline Cepas & $\begin{array}{l}\text { Crecimiento } \\
\text { en AM }\end{array}$ & Micelio aéreo & $\begin{array}{l}\text { Reverso de } \\
\text { colonia }\end{array}$ & $\begin{array}{l}\text { Origen } \\
\text { (bahía) }\end{array}$ \\
\hline B1-CD1 & + & Blanco & Amarillo & I \\
B1-IM2 & ++ & Blanco & Amarillo & I \\
B1TG1 & +++ & Blanco & Amarillo & I \\
B1-662 & +++ & Blanco & Amarillo & I \\
II334C & ++ & Gris & Amarillo & I \\
M10-77 & +++ & Blanco & Rojo & I \\
M10-85 & ++ & Blanco & Amarillo & I \\
M11-105 & ++ & Blanco & Amarillo & I \\
M11-106 & ++ & Blanco & Amarillo & I \\
M11-111 & ++ & Blanco & Amarillo & I \\
M11-115 & + & Blanco & Amarillo & I \\
M11-116 & ++ & Gris & Amarillo & I \\
M11-116a(2) & +++ & Gris & Marrón & I \\
M11-116b(2) & - & - & - & I \\
M11-116(B) & +++ & Gris & Marrón & I \\
M11-116d & ++ & Gris & Amarillo & I \\
M11 116d(A) & ++ & Gris & Marrón & I \\
M11-116d(B) & ++ & Gris & Marrón & I \\
M11-133 & ++ & Blanco & Amarillo & I \\
I300A(2) & +++ & Gris & Amarillo & A \\
I434Bb & - & - & - & A \\
I434B & +++ & Blanco & Marrón & A \\
I434Bc & +++ & Blanco & Amarillo & A \\
E54Ba & +++ & Blanco & Marrón & A \\
\hline
\end{tabular}

Crecimiento en Agar Marino (AM): +++: Abundante; ++: moderado; +: escaso; -: no crecimiento I: Bahía de Independencia; A: Bahía de Ancón

patogenia; sin embargo, otros reportes señalan la alta virulencia de estas especies de Vibrio en langostinos (Peña-Navarro y Varela-Mejías, 2015). El aislamiento de Vibrio a partir del hepatopáncreas, hemolinfa y tracto digestivo de $L$. vannamei fue reportado por Suárez (2008) con resultados similares al presente estudio, donde la mayor parte fueron fermentadores de sacarosa en el medio TCBS, mientras que, en este caso, solo la cepa V3L fue aislada de hemolinfa e identificada como $V$. alginolyticus.
El metabolismo de Vibrio siempre fue complejo; tal es así que algunos resultados de las pruebas bioquímicas difieren respecto a los patrones, tal como ocurre con las cepas V7P3, V8P1 y V8P2 del presente estudio, que resultaron ser citrato-positivas. Esta variación es muy frecuente en el comportamiento de cepas ambientales de Vibrio (Ganesh et al., 2010). Asimismo, se han evidenciado problemas en la identificación por el sistema miniaturizado API, ya que suelen presentarse muchas discordancias con los resultados 
Cuadro 5. Actividad enzimática extracelular de actinomicetos marinos a diversos sustratos

\begin{tabular}{|c|c|c|c|c|c|c|c|}
\hline \multirow{2}{*}{ Cepas } & \multicolumn{4}{|c|}{ Halos de hidrólisis (mm) } & \multirow{2}{*}{ DNAsa } & \multirow{2}{*}{ Gelatinasa } & \multirow{2}{*}{ Ureasa } \\
\hline & Amilasa & Esterasa & Caseinasa & Lecitinasa & & & \\
\hline B1-CD1 & 44 & 19.8 & 17 & 9.1 & - & - & - \\
\hline B1-IM2 & 45 & 8.8 & 0 & 0 & + & +++ & + \\
\hline B1TG1 & 20.6 & 23.4 & 52.3 & 16.4 & + & +++ & - \\
\hline B1-662 & 12 & 0 & 25 & 20.5 & - & +++ & - \\
\hline II334C & 10.6 & 19 & 0 & 0 & - & + & - \\
\hline M10-77 & 24 & 0 & 0 & 14 & + & + & + \\
\hline M10-85 & 0 & 0 & 0 & 9.5 & - & ++ & - \\
\hline M11-105 & 28 & 12 & 0 & 0 & + & +++ & + \\
\hline M11-106 & 42 & 8 & 7 & 7.4 & - & +++ & + \\
\hline M11-111 & 0 & 0 & 0 & 16.9 & - & ++ & - \\
\hline M11-115 & 29 & 0 & 0 & 17.9 & - & +++ & - \\
\hline M11-116a & 25 & 0 & 21 & 14.3 & - & ++ & + \\
\hline $\begin{array}{l}\text { M11- } \\
116 a(2)\end{array}$ & 36 & 29.3 & 23 & 0 & - & + & - \\
\hline $\begin{array}{l}\text { M11- } \\
116 \mathrm{~b}(2)\end{array}$ & 0 & 0 & 0 & 0 & - & + & - \\
\hline $\begin{array}{l}\text { M11- } \\
116(\mathrm{~B})\end{array}$ & 33 & 21.3 & 40 & 0 & - & + & - \\
\hline M11-116d & 33 & 20 & 19 & 17.6 & + & + & + \\
\hline $\begin{array}{l}\text { M11 } \\
116 \mathrm{~d}(\mathrm{~A})\end{array}$ & 43.1 & 19.3 & 30.6 & 16.6 & - & + & - \\
\hline $\begin{array}{l}\text { M11- } \\
116 \mathrm{~d}(\mathrm{~B})\end{array}$ & 32.1 & 18 & 25.6 & 0 & - & + & - \\
\hline M11-133 & 0 & 28.5 & 0 & 15.5 & + & ++ & + \\
\hline $\mathrm{I} 300 \mathrm{~A}(2)$ & 18 & 0 & 15.5 & 0 & - & + & - \\
\hline $\mathrm{I} 434 \mathrm{Bb}$ & 0 & 0 & 0 & 0 & - & ++ & - \\
\hline I434B & 40 & 17.6 & 24 & 15.8 & + & +++ & + \\
\hline $\mathrm{I} 434 \mathrm{Bc}$ & 21 & 0 & 14 & 16.6 & - & + & + \\
\hline E54Ba & 55.5 & 14.6 & 41.6 & 22.6 & + & +++ & - \\
\hline
\end{tabular}

Agar DNA y Agar Urea: positiva (+), negativa (-)

Agar Gelatina: hidrólisis total (+++), parcial (++), moderada (+), no hidrólisis (-)

obtenidos mediante técnicas bacteriológicas convencionales (Orozco et al., 2017).

El cultivo de langostinos por ser una actividad intensiva ha requerido del uso de antimicrobianos para el control de patógenos (Kümmerer, 2009), pero esta actividad es actualmente cuestionada por ser responsa- ble de la resistencia bacteriana a los antibióticos. En el presente estudio, los resultados señalan que todas las cepas de Vibrio fueron sensibles al ciprofloxacino y al enroblend, aunque resistentes a novobiocina y penicilina $G$, en tanto que dos de las cepas mostraron resistencia al florfenicol y al ácido nalidíxico. Estos datos concuerdan con el es- 
Cuadro 6. Características de cultivo de cinco actinomicetos en diferentes medios estándar ISP

\begin{tabular}{|c|c|c|c|c|c|}
\hline \multirow[t]{2}{*}{ Cepa } & \multirow{2}{*}{ Medio } & \multicolumn{2}{|c|}{ Color del micelio } & \multirow{2}{*}{$\begin{array}{l}\text { Pigmento } \\
\text { soluble }\end{array}$} & \multirow{2}{*}{ Crecimiento } \\
\hline & & Aéreo & Lado reverso & & \\
\hline \multirow[t]{6}{*}{ M10-77 } & ISP2 & gris & marrón claro & ninguno & ++ \\
\hline & ISP3 & gris & marrón rojizo & verde & ++ \\
\hline & ISP4 & gris & gris & ninguno & ++ \\
\hline & ISP5 & marrón & gris & ninguno & + \\
\hline & ISP6 & beige & amarillo & ninguno & + \\
\hline & ISP7 & gris & marrón oscuro & ninguno & ++ \\
\hline \multirow[t]{6}{*}{ M11-116(B) } & ISP2 & gris & marrón claro & ninguno & ++ \\
\hline & ISP3 & gris & amarillo & ninguno & ++ \\
\hline & ISP4 & gris & marrón claro & gris & ++ \\
\hline & ISP5 & blanco & blanco & ninguno & + \\
\hline & ISP6 & beige & amarillo & ninguno & + \\
\hline & ISP7 & blanco & marrón claro & gris & ++ \\
\hline \multirow[t]{6}{*}{ M11-116d(B) } & ISP2 & gris & amarillo & ninguno & ++ \\
\hline & ISP3 & gris & gris & ninguno & ++ \\
\hline & ISP4 & blanco & marrón claro & gris & ++ \\
\hline & ISP5 & blanco & blanco & ninguno & + \\
\hline & ISP6 & beige & beige & ninguno & + \\
\hline & ISP7 & blanco & marrón oscuro & gris & ++ \\
\hline \multirow[t]{6}{*}{$\mathrm{I} 300 \mathrm{~A}$} & ISP2 & gris & marrón rojizo & ninguno & ++ \\
\hline & ISP3 & gris & marrón claro & marrón & ++ \\
\hline & ISP4 & gris & gris & ninguno & ++ \\
\hline & ISP5 & blanco & amarillo & amarillo & + \\
\hline & ISP6 & blanco & amarillo & ninguno & + \\
\hline & ISP7 & gris & marrón oscuro & gris & ++ \\
\hline \multirow[t]{6}{*}{ M11-116d(A) } & ISP2 & blanco & marrón oscuro & ninguno & ++ \\
\hline & ISP3 & gris & gris & ninguno & ++ \\
\hline & ISP4 & gris & gris & ninguno & ++ \\
\hline & ISP5 & blanco & blanco & ninguno & + \\
\hline & ISP6 & beige & amarillo oscuro & ninguno & + \\
\hline & ISP7 & gris & marrón oscuro & gris & ++ \\
\hline
\end{tabular}

Crecimiento: ++: Moderado; +: escaso

ISP2 (Agar extracto de levadura - extracto de malta)

ISP3 (Agar avena)

ISP4 (Agar sales inorgánicas - almidón)

ISP5 (Agar glicerol - asparagina)

ISP6 (Agar peptona hierro - extracto de levadura)

ISP7 (Agar tirosina) 
Cuadro 7. Antagonismo de actinomicetos marinos frente a cepas de Vibrio aislados de $L$. vannamei (expresados en $\mathrm{mm}$ de halos de inhibición)

\begin{tabular}{|c|c|c|c|c|c|c|c|c|c|}
\hline \multirow{2}{*}{ Cepas } & \multicolumn{6}{|c|}{ Cepas silvestres } & \multicolumn{3}{|c|}{ Cepas control } \\
\hline & V8P3 & V5P3 & V3L & V8P2 & $\mathrm{V} 8 \mathrm{P} 1$ & V7P3 & VA & VP & $\mathrm{VH}$ \\
\hline B1-CD1 & 1.8 & 0 & 0 & 0 & 0 & 0 & 0 & NR & 3.5 \\
\hline B1-IM2 & 7 & 0 & 0 & 0 & 0 & 0 & 0 & NR & 0 \\
\hline B1TG1 & 7.4 & 0 & 0 & 13.3 & 10.2 & 14.3 & 0 & NR & $\mathrm{NC}$ \\
\hline B1-662 & 0 & 0 & 0 & 0 & 0 & 0 & 0 & NR & 0 \\
\hline II $334 \mathrm{C}$ & 28.4 & 9.7 & 3 & 10.3 & 10.6 & 11 & 6 & 13.5 & $\mathrm{NC}$ \\
\hline M10-77 & 21 & 10.9 & 14.6 & 21.2 & 19.1 & 21.5 & 17 & 18.9 & 15.8 \\
\hline M10-85 & 0 & 0 & 0 & 0 & 0 & 0 & 0 & NR & 0 \\
\hline M11-105 & 0 & 0 & 0 & 0 & 0 & 0 & 3.5 & NR & 0 \\
\hline M11-106 & 0 & 0 & 0 & 0 & 0 & 0 & 0 & NR & 4 \\
\hline M11-111 & 8.5 & 0 & 0 & 0 & 0 & 0 & 0 & NR & 0 \\
\hline M11-115 & 0 & 0 & 0 & 0 & 0 & 0 & 6.5 & NR & 0 \\
\hline M11-116a & 4.25 & 6 & 8.5 & 0 & 5 & 5.1 & 0 & NR & 0 \\
\hline M11-116a(2) & 16.4 & 12.1 & 20.2 & 16.9 & 6.5 & 0 & 0 & 5.8 & 8.8 \\
\hline M11-116b(2) & 0 & 0 & 0 & 0 & 0 & 0 & $\mathrm{NC}$ & $\mathrm{NC}$ & $\mathrm{NC}$ \\
\hline M11-116(B) & 19.3 & 19.1 & 18.9 & 14.7 & 16.8 & 14.8 & 18.5 & 16.5 & 7.7 \\
\hline M11-116d & 0 & 2.6 & 2 & 1.5 & 0 & 6.1 & 0 & NR & 2 \\
\hline M11 116d(A) & 18.9 & 20.1 & 18.8 & 18.9 & 10.8 & 8.3 & 0 & 17.8 & 10.3 \\
\hline M11-116d(B) & 22.8 & 10.3 & 18.2 & 15.3 & 15.9 & 12.8 & 10.3 & NR & $\mathrm{NC}$ \\
\hline M11-133 & 0 & 0 & 0 & 0 & 0 & 0 & 0 & NR & 0 \\
\hline $\mathrm{I} 300 \mathrm{~A}(2)$ & 3.8 & 0 & 10.5 & 9.3 & 4.2 & 3.8 & 14 & NR & 0 \\
\hline $\mathrm{I} 434 \mathrm{Bb}$ & 0 & 0 & 0 & 0 & 0 & 0 & 0 & 0 & 0 \\
\hline I434B & 0 & 0 & 0 & 6.3 & 0 & 8.7 & 8.7 & 0 & 4.2 \\
\hline $\mathrm{I} 434 \mathrm{Bc}$ & 0 & 0 & 0 & 0 & 0 & 0 & 0 & NR & 5 \\
\hline $\mathrm{E} 54 \mathrm{Ba}$ & 1.4 & 16.1 & 0 & 6.5 & 5 & 5 & 7.1 & 9.2 & 5.5 \\
\hline
\end{tabular}

NC: No creció; NR: no se realizó

VA: V. alginolyticus; VP: V. parahaemolyticus; VH: V.harveyi

tudio de Dos Santos et al. (2016), quienes encontraron que las 70 cepas de Vibrio resultaron ser sensibles al ciprofloxacino, aunque 33 fueron resistentes al florfenicol. Cabe resaltar que la cepa de Vibrio V8P3 mostró resistencia a florfenicol, un antibiótico usado en la industria acuícola.

En el estudio de actinomicetos, el color del micelio aéreo y del sustrato es considerado importante para la agrupación y la identi- ficación de géneros, particularmente para Streptomyces. Por ello, la caracterización en los medios ISP resulta de mucha ayuda, tal como fue señalado también en otros reportes (Mohammd y Haidar, 2014). La identificación convencional de Streptomyces se basa en estudios morfológicos y microscópicos, particularmente en la pigmentación soluble de color marrón, rojo o amarillo en los medios de cultivo ISP (Shirling y Gottlieb, 1966); sin embargo, cabe resaltar que las característi- 
Cuadro 8. Porcentajes de homología de cepas de Vibrio aislados de L. vannamei

\begin{tabular}{cl}
\hline & \multicolumn{1}{c}{ Cepas } \\
\hline V3L & Vibrio alginolyticus / V. parahaemolyticus $(100 \%)$ \\
V5P3 & Vibrio alginolyticus /V. parahaemolyticus $(100 \%)$ \\
V7P3 & Vibrio sp. $(98 \%)$ \\
V8P1 & Vibrio diabolicus / V. mytili $(100 \% / 99 \%)$ \\
V8P2 & Vibrio diabolicus /V. mytili $(99 \%)$ \\
V8P3 & Vibrio parahaemolyticus / V. diabolicus $(100 \%)$ \\
\hline
\end{tabular}

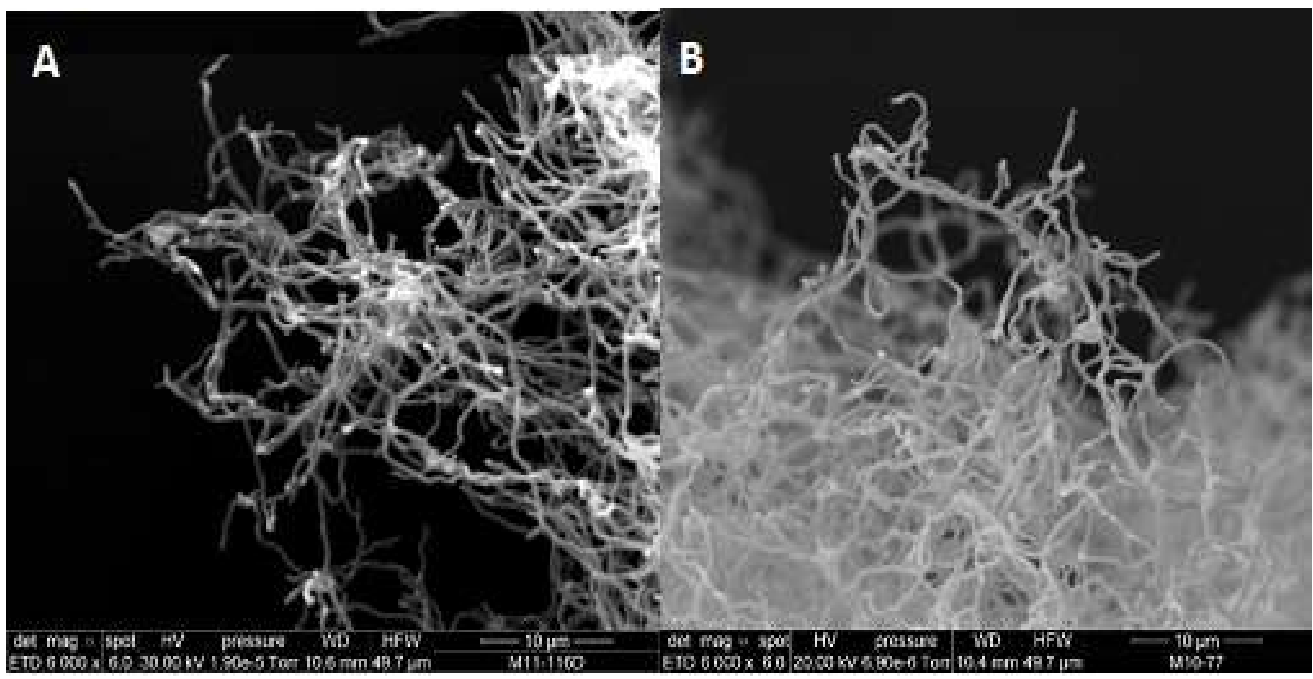

Figura 1. Microfotografía de actinomicetos. A: cepa M11-116d. B: cepa M10-77. 6000X

cas fisiológicas suelen variar con los nutrientes disponibles y condiciones físicas donde se desarrollan (Vijayakumar et al., 2010).

Trabajos previos de León et al. (2007) señalan que los actinomicetos de sedimentos marinos son grandes productores de EEC como la caseinasa, tween esterasa y lecitinasa. Los resultados concuerdan parcialmente con lo reportado para las 24 cepas del estudio, donde se resalta la actividad proteolítica sobre la gelatina (96\%), seguido por la actividad amilolítica ( $80 \%$ ) y lipolítica sobre lecitina (64\%). En otro reporte, León et al. (2016) señalan a la gelatinasa (81\%) como la EEC más abundante en actinomicetos aislados de Argopecten purpuratus.

En un estudio de antagonismo similar al presente, entre actinomicetos aislados del sedimento de estanques de langostinos (Penaeus monodon) moribundos y Vibrio aislado del músculo e hígado del mismo organismo, se obtuvo halos de inhibición hasta un máximo de $40 \mathrm{~mm}$ de diámetro (Chau et al., 


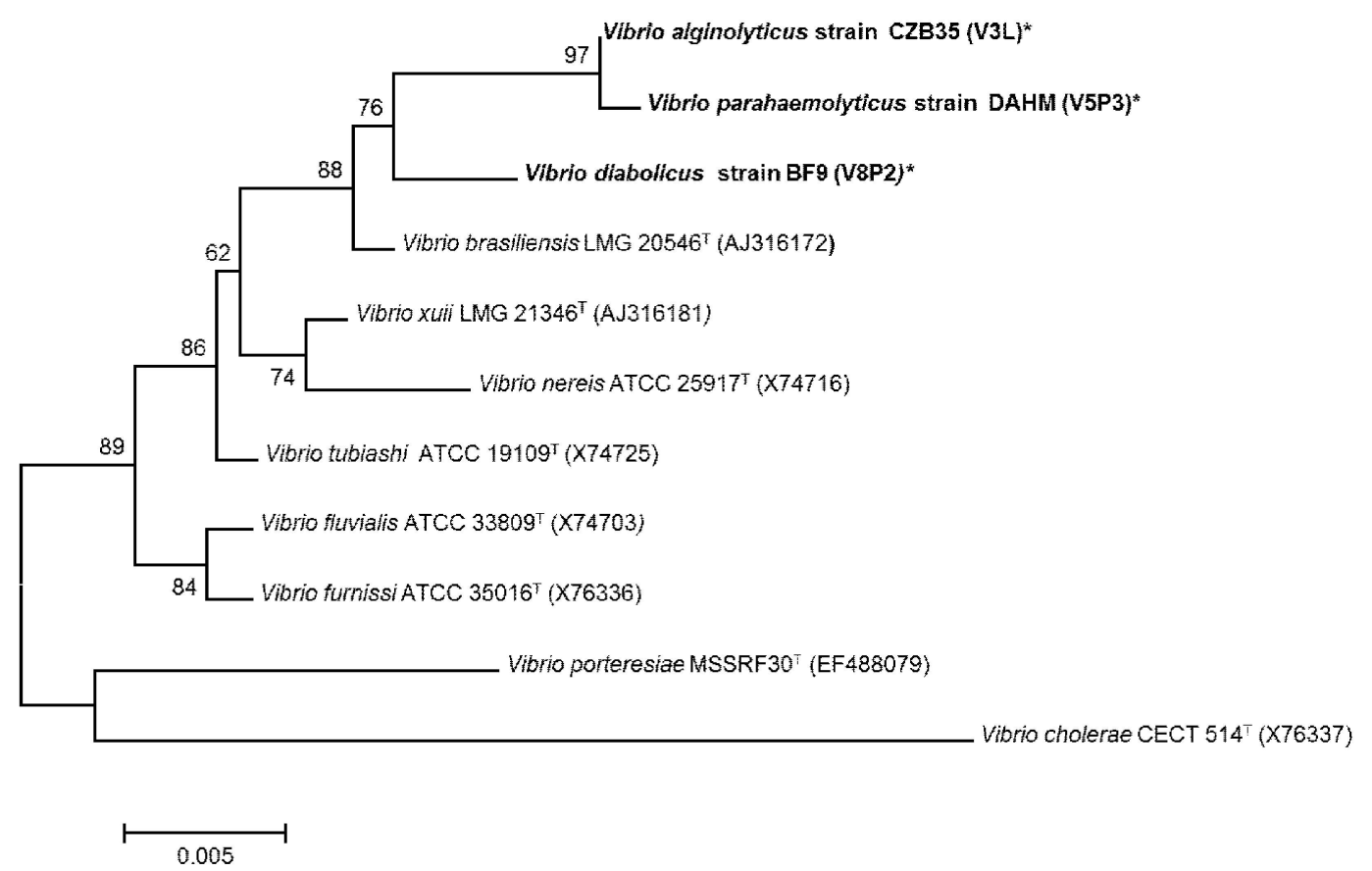

Figura 2. Árbol filogenético según el método Neighbor-Joining basado en la secuencia del gen 16S ARNr de cultivos de las cepas de Vibrio V8P2, V5P3 y V3L. En cada rama se muestran los valores de bootstrap (500 repeticiones) presentando valores $\geq 50 \%$. El árbol se construyó usando como base las secuencias de Vibrio porteresiae y Vibrio cholerae. La barra de la escala representa 0.005 sustituciones por posición de base. En negrita las cepas obtenidas en este estudio

2011). Estos resultados son comparables al presente estudio donde se obtuvo halos con valores máximos de $28.4 \mathrm{~mm}$ frente al Vibrio sp. V8P3 (identificado como Vibrio diabolicus). El método de «doble capa» resultó útil en la selección de actinomicetos con actividad anti-Vibrio.

You et al. (2005) aislaron 94 actinomicetos de langostinos en China, donde el $51 \%$ inhibió a cepas de Vibrio, pero $24.5 \%$ inhibió a siete especies de Vibrio patógenas de peces y langostinos. La mayoría de estos antagonistas resultaron ser Streptomyces. Por otro lado, Das et al. (2010) determinaron la actividad antimicrobiana de 43 actinomicetos aislados en una langostinera en Australia frente a especies de Vibrio, encontrando que 12 tenían actividad anti-Vibrio, 2 fueron anta- gonistas a 5 patógenos y solo 8 lograron inhibir a $V$. harveyi (considerado el principal patógeno de langostinos). Por su parte, León et al. (2016), en un trabajo similar, reportaron que $37 \%$ de actinomicetos aislados de Argopecten purpuratus presentaban actividad anti Vibrio frente a $V$. vulnificus, $V$. anguillarum y $V$. alginolyticus. En el presente estudio, el $83.3 \%$ (20) de las 24 cepas probadas logró presentar actividad inhibitoria frente a alguna de las cepas de Vibrio probadas y el 8.3\% (2) logró inhibir a las 10 cepas de Vibrio, encontrando, además, que 3 cepas lograban inhibir a $V$. harveyi, $V$. parahaemolyticus y $V$. alginolyticus.

Las cepas de Vibrio aisladas de $L$. vannamei y las cepas control tipificadas como patógenas de peces, presentan valores de in- 


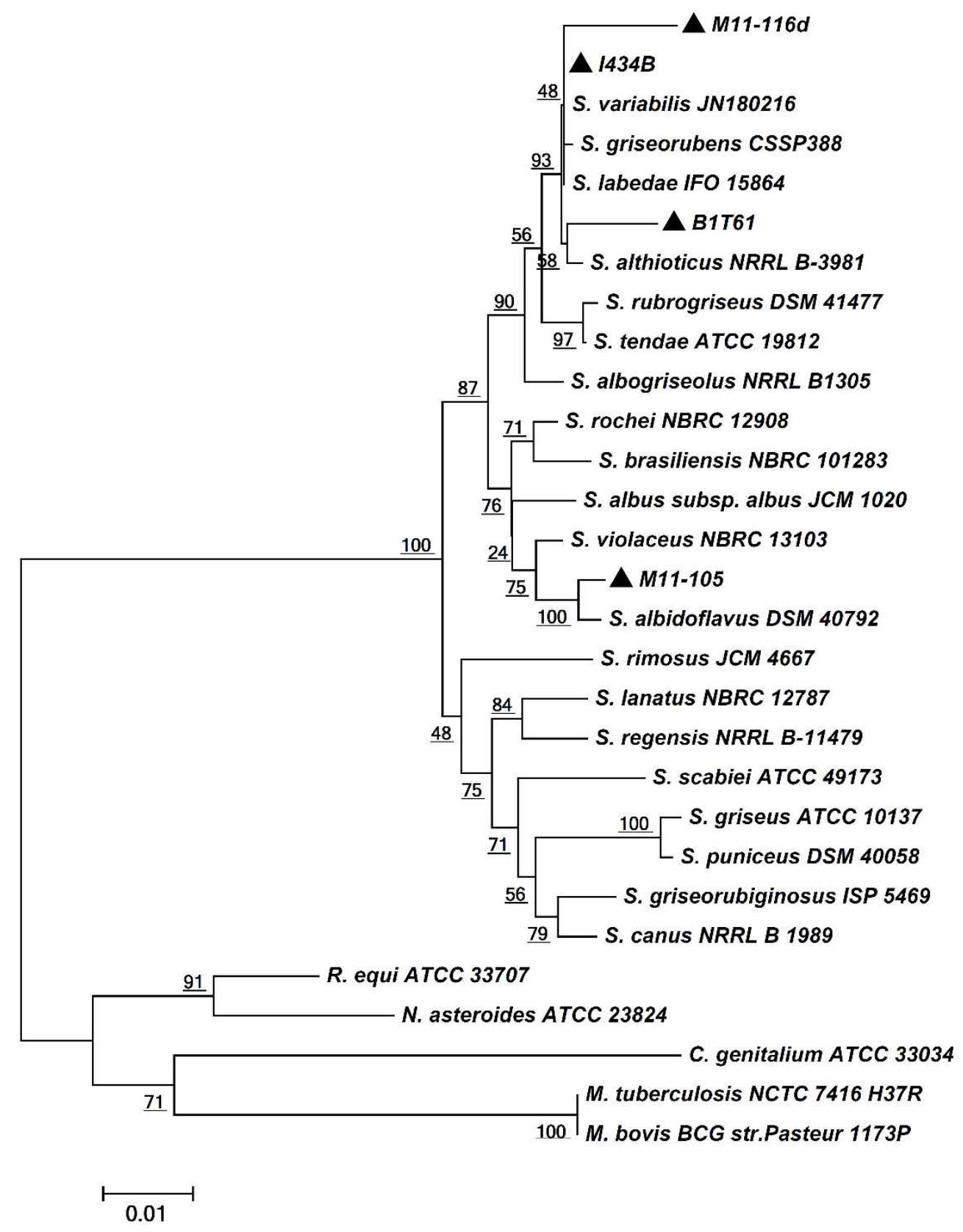

Figura 3.Árbol filogenético según el método Neighbor-Joining basado en la secuencia del gen 16S ARNr de cepas aisladas de sedimento marino (M11-116d, I434B, B1T61 y M11105) y especies relacionadas del género Streptomyces. Los números internos de los nodos corresponden a los valores de soporte de bootstrap $\geq 45 \%$ (1000 repeticiones). Las secuencias de los géneros Corynebacterium, Rhodococcus, Nocardia y Mycobacterium fueron elegidas por su relación filogenética cercana con el género Streptomyces (barra, 0.01 sustituciones por posición de base) 
hibición similares. Cabe resaltar que las cepas silvestres siempre resultan más difíciles de inhibir debido a mecanismos de resistencia que pueden haber adquirido de su medio ambiente natural (Santiago et al., 2009).

Las pruebas moleculares de identificación resultaron ser muy útiles tanto para el género Vibrio como para los actinomicetos. Aún no existen datos en el país sobre el aislamiento de $V$. diabolicus, siendo este el único reporte de esta especie asociada a $L$. vannamei. En Brasil, Dos Santos et al. (2016) aislaron 70 cepas de Vibrio de un estuario donde se cultiva $L$. vannamei logrando identificar tres cepas como $V$. diabolicus, las cuales fueron aisladas del agua y sedimento. Respecto a la identificación molecular de Streptomyces, cabe señalar que las especies de $S$. variabilis, S. griseorubens, $S$. althioticus y $S$. albidoflavus han sido reportadas en sedimento marino (Sudha et al., 2015; Shubha et al., 2017). Estas especies, dada la eficacia de las pruebas in vitro frente a patógenos marinos, han sido planteadas como potenciales candidatos a probióticos (Tan et al., 2016).

Investigaciones recientes han demostrado el potencial de Streptomyces marinos en acuicultura. Estos microorganismos pueden producir diversos metabolitos bioactivos (Manivasagan et al., 2013), que pueden actuar como agentes de biocontrol de cepas patógenas de Vibrio, desarrollar actividad inhibitoria de virus y promover el desarrollo intensivo (Kumar et al., 2016). Aún quedan muchas interrogantes por responder; sin embargo, todo indica que los actinomicetos marinos son grandes productores de metabolitos secundarios con diversos efectos benéficos para su aplicación en actividades de la acuicultura.

\section{Conclusiones}

Los resultados del presente trabajo permiten señalar que los actinomicetos de sedimento marino, principalmente del género
Streptomyces conforman un grupo selecto de microorganismos ambientales con múltiples potencialidades. Gracias a su amplia y compleja capacidad metabólica y su marcado antagonismo frente a representantes patógenos del género Vibrio pueden ser considerados como potenciales cepas probióticas en el cultivo de langostinos; sin embargo, se requiere mayores investigaciones al respecto.

\section{Agradecimientos}

Al Vicerrectorado de Investigación y Posgrado (VRIP) de la Universidad Nacional Mayor de San Marcos, Lima - Perú, por el financiamiento parcial a través del Estudio de Investigación CON CON 2013 (R.R. N. ${ }^{\circ}$ 00690-R-13).

\section{Literatura Citada}

1. Altschup SF, Gish W, Miller W, Myers EW, Lipman DJ. 1990. Basic local alignment search tool. J Molec Biol 215: 403-410. doi: 10.1016/S0022-2836(05)80360-2

2. Baumann P, Schubert RHW. 1984. Family II. Vibrionaceae Veron 1965. In: Krieg NR, Holt JG (eds). Bergey's Manual of systematic bacteriology. Vol 2. Baltimore: Williams \& Wilkins. p516-517.

3. Chau N, Hieu N, Thuan L, Matsumoto M, Miyajima I. 2011. Identification and characterization of actinomycetes antagonistic to Vibrio spp isolated from shrimp culture pond in Thua Thien Hue - Viet Nam. J Fac Agric Kyushu Univ 56(1): 15-22.

4. Colona E, Galindo N, León J, Alzamora L. 2014. Desarrollo de un método para la observación de actinomicetos por microscopía electrónica de barrido. En: XXIII Reunión Científica ICBAR. Lima, Perú.

5. Das S, Ward LR, Burke C. 2010. Screening of marine Streptomyces spp for potential use as probiotics in aquaculture. Aquaculture 305: 32-41. doi: 10.1016/j.aquaculture.2010.04.001 
6. Dos Santos R, Viana O, Silva R. 2016. Multidrug-resistant Vibrio associated with an estuary affected by shrimp farming in Northeastern Brazil. Mar Poll Bull 105: 337-340. doi: 10.1016/ j.marpolbul.2016.02.001

7. Ganesh EA, Das S, Chandrasekar K, Arun G, Balamurugan S. 2010. Monitoring of total heterotrophic bacteria and Vibrio spp in an aquaculture pond. Curr Res J Biol Sci 2(1): 48-52.

8. [INS] Instituto Nacional de Salud. 2002. Manual de procedimientos para la prueba de sensibilidad antimicrobiana por el método de disco difusión. Serie de Normas Técnicas N. ${ }^{\circ}$ 30. Lima, Perú: INS. $67 \mathrm{p}$.

9. Jukes TH, Cantor CR. 1969. Evolution of protein molecules. In: Munro HN (ed). Mammalian protein metabolism. New York: Academic Press. p 21-132.

10. Kumar S, Philip R, Achuthankutty $C$. 2016. Antiviral property of marine actinomycetes against white spot syndrome virus in penaeid shrimps. Curr Sci 91: 807-811.

11. Kümmerer K. 2009. Chemosphere antibiotics in the aquatic environment a review - Part I. Chemosphere 75: 417434. doi: 10.1016/j.chemosphere.2008.11.086

12. Larkin MA, Blackshields G, Brown NP, Chenna R, McGettigan PA, McWilliam H, Valentin F, et al. 2007. Clustal W and Clustal X version 2.0. Bioinformatics 23: 2947-2948. doi: 10.1093/bioinformatics/btm404

13. León J, Aponte JJ, Cuadra DL, Galindo N, Jaramillo L, Vallejo M, Marguet E. 2016. Actinomicetos aislados de Argopecten purpuratus productores de enzimas extracelulares y con actividad inhibitoria de patógenos marinos. Rev Biol Mar Ocean 51: 69-80. doi: 10.4067/S0718-19572016000100007

14. León J, Liza L, Soto I, Cuadra DL, Patiño L, Zerpa R. 2007. Actinomicetos bioactivos de sedimento marino de la costa central del Perú. Rev Per Biol 14: 259-270.
15. Liu H, Li Z, Tan B, Lao Y, Duan Z, Sun W, Dong $X$. 2014. Isolation of a putative probiotic strain S12 and its effect on growth performance, non-specific immunity and disease-resistance of white shrimp, Litopenaeus vannamei. Fish Shellfish Immunol 41: 300-307. doi: 10.1016/j.fsi.2014.08.028

16. Manivasagan $P$, Venkatesan $J$, Sivakumar K, Kim SK. 2013. Marine actinobacterial metabolites: Current status and future perspectives. Microbiol Res 168: 311-332. doi: 10.1016/ j.micres.2013.02.002

17. Mohammd E, Haidar N. 2014. Extraction and purification of antimicrobial agent produced from actinomycetes isolated from agriculture soils. $\mathrm{J}$ Babyl Univ/Pure Appl Sci 22(2): 1-10.

18. Nimrat S, Suksawat S, Boonthai T, Vuthiphandchai V. 2012. Potential Bacillus probiotics enhance bacterial numbers, water quality and growth during early development of white shrimp (Litopenaeus vannamei). Vet Microbiol 159: 443-450. doi: 10.1016/j.vetmic.2012.04.029

19. Orozco R, Quispe Y, Lorenzo A, Zamudio ML. 2017. Asociación de floraciones de algas nocivas y Vibrio spp en áreas de pesca y acuicultura de bivalvos de moluscos en las bahías de Sechura y Pisco, Perú. Rev Perú Biol 24: 111-116. doi: 10.15381/rpb.v24i1.13111

20. Peña-Navarro N, Varela Mejías $A$. 2015. Análisis histopatológico en Litopenaeus vannamei infectado con Vibrio parahaemolyticus. Agron Mesoam 26: 43-53. doi: 10.15517/am.v26i1.16892

21. Santiago M, Espinosa A, Bermudez M del C. 2009. Uso de antibióticos en la camaronicultura. Rev Mex Cienc Farm 40(3): 22-32.

22. Shirling E, Gottlieb D. 1966. Methods for characterization of Streptomyces species. Int J Syst Bacteriol 16: 313-340. doi: 10.1099/00207713-16-3-313 
23. Shubha M, Ushashi B, Veena S, Bhaskara R. 2017. Antimicrovial activity of Streptomyces variabilis strain VITUMVB03 isolated from Kanyakumari marine sediments. Asian J Pharm Clin Res 10: 112-116.

24. Suarez C. 2008. Cuantificación y caracterización molecular de bacterias de hemolinfa de camarones Litopenaeus vannamei durante brotes del síndrome de mancha blanca y evaluación de sensibilidad a cinco productos antibacterianos. Tesis de Microbiólogo Industrial. Bogotá, Colombia: Pontificia Universidad Javeriana. $230 \mathrm{p}$.

25. Sudha Y, Lakhsmi H, Sharmila S. 2015. Isolation, screening, identification, characterization and application of green synthesized silver nanoparticle from marine actinomycetes Streptomyces althioticus. World J Pharm Res 4: 15921611.

26. Tamura K, Stecher G, Peterson D, Filipski A, Kumar S. 2013. MEGA6: Molecular Evolutionary Genetics Analysis version 6.0. Molec Biol Evol 30: 2725-2729. doi: 10.1093/molbev/mst197

27. Tan LT, Chan K, Lee L, Goh B. 2016. Streptomyces bacteria as potential probiotics in aquaculture. Front Microbiol 7: 79. doi: 10.3389/fmicb.2016.00079

28. Vaseeharan B, Ramasamy $P$, Murugan T, Chen JC. 2005. In vitro susceptibility of antibiotics against Vibrio spp and Aeromonas spp isolated from Penaeus monodon hatcheries and ponds. Int J Antimicrob Agent 26: 285291. doi: 10.1016/j.ijantimicag.2005.07.005
29. Verschuere L, Rombaut G, Sorgeloos $P$, Verstraete $W$. 2000. Probiotic bacteria as biological control agents in aquaculture. Microbiol Molec Biol Rev 64: 655-671. doi: 10.1128/MMBR.64.4.655-671.2000

30. Vijayakumar $R$, Murugesan $S$, Panneerselvam A. 2010. Isolation, characterization and antimicrobial activity of actinobacteria from Point Calimere coastal region, East Coast of India. Int Res J Pharm 1: 358-365.

31. Wang D, Xue Q, Ma Y, Wei X, Chen J, He F. 2014. Oligotrophy is helpful for the isolation of bioactive Actinomycetes. Ind J Microbiol 54: 178184. doi: 10.1007/s12088-014-0444-1

32. Yano Y, Hamano K, Tsutsui I, AueUmneoy D, Ban M, Satomi M. 2015. Occurrence, molecular characterization, and antimicrobial susceptibility of Aeromonas spp in marine species of shrimps cultured at inland low salinity ponds. Food Microbiol 47: 21-27. doi: 10.1016/j.fm.2014.11.003

33. You JL, Cao LX, Liu GF, Zhou SN, Tan HM, Lin YC. 2005. Isolation and characterization of actinomycetes antagonistic to pathogenic Vibrio spp from nearshore marine sediments. World J Microbiol Biotech 21: 679-682. doi: 10.1007/s11274-004-3851-3

34. Zhou J, Fang $W$, Yang $X$, Zhou $S$, Hu $L, L i X, Q i X .2012$. A nonluminescent and highly virulent Vibrio harveyi strain is associated with «bacterial white tail disease» of Litopenaeus vannamei Shrimp. PLoS ONE 7: 1-6. doi: 10.1371/ journal.pone.0029961 